\title{
Genes Up-Regulated in Tolerant Cavendish Banana Roots in Response to Fusarium oxysporum f. sp. cubense Infection ${ }^{1}$
}

N. van den Berg ${ }^{1}$, D.K. Berger ${ }^{2}$, I. Hein ${ }^{3}$, P.R.J. Birch ${ }^{3}$, M.J. Wingfield ${ }^{1}$ and A. Viljoen ${ }^{1}$

${ }^{1}$ Department of Microbiology and Plant Pathology, Forestry and Agricultural Biotechnology Institute (FABI), University of Pretoria, 0002, South Africa

2 Department of Botany, Forestry and Agricultural Biotechnology Institute (FABI), University of Pretoria, 0002, South Africa

3 Plant Pathology Programme, Scottish Crop Research Institute, Invergowrie, Dundee, DD2 5DA, UK

Keywords: catalase, defence-associated genes, $P A E, P R-1, P R-3$, phenolic compounds

\begin{abstract}
Fusarium wilt of banana, caused by Fusarium oxysporum f. sp. cubense (Foc), is regarded as one of the most devastating diseases. Cavendish cultivars (AAA genome) are highly susceptible to race 4 of the pathogen. The regulation of defencerelated genes in 'GCTCV-218', a Fusarium wilt-tolerant Cavendish selection, was studied. A cDNA library, enriched for genes up-regulated in 'GCTCV-218' after Foc infection when compared to susceptible Cavendish cultivar 'Williams', was previously constructed using suppression subtractive hybridisation (SSH) and screened using DNA micro-array technology. Potentially induced clones (334) were selected and sequenced. Four defence-associated genes (catalase 2, pectin acetyl esterase $(P A E), P R-1$ and $P R-3)$ were selected for expression profile analysis using real-time reverse transcriptase PCR (RT-PCR). 'GCTCV-218' showed significantly lower disease severity and incidence than the susceptible 'Williams' in replicated greenhouse and field trials. Several defence-related transcripts were identified by BLASTX searches, including genes coding for PR-1, PAE, xylanase inhibitor, peroxidase, catalase 2, metallothionein, response regulator 6 and tripsin inhibitor. Inverse Northern dot blot data indicated that all of the above-mentioned transcripts were up-regulated in 'GCTCV-218' 6 hours post inoculation (hpi) compared to 'Williams'. Quantitative RT-PCR results confirmed that all four genes were differentially expressed in 'GCTCV-218' 3 and 6 hpi, confirming SSH results. PR-1 and $P A E$ were induced very early ( $3 \mathrm{hpi})$ in 'GCTCV-218', while $P R-3$ and catalase 2 followed with a significant induction $6 \mathrm{hpi}$. This study concluded that ' $G C T C V-218$ ' is able to respond rapidly to Foc infection by activating both a biochemical and structural defence mechanism. The tolerance of 'GCTCV-218' was further linked to a significant increase in the induction cell wall-associated phenolic compounds.
\end{abstract}

\section{INTRODUCTION}

Fusarium wilt of banana, caused by the soil-borne pathogen Fusarium oxysporum f. sp. cubense (Foc), is regarded as one of the most serious diseases and has destroyed many thousands of hectares of Cavendish (AAA genome) bananas in tropics (Hwang and Ko, 2004) and subtropics (Viljoen, 2002). No sustainable control strategy exists, other than replacing susceptible cultivars with those resistant to the disease. In the mid-1900s, race 1 of the pathogen led to the replacement of the susceptible 'Gros Michel' (AAA genome) with resistant Cavendish cultivars as the main banana for export. However, Cavendish cultivars are highly susceptible to Foc race 4 . There is no commercial cultivar with resistance to Foc race 4 available to replace Cavendish. Natural disease resistance exists in wild-type bananas, but these are not suitable for the consumer market. Conventional breeding efforts to find a tolerant or resistant replacement have had limited success due to the fact that Cavendish cultivars are parthenocarpic (Robinson, 1996). Hwang and Tang (1996) therefore initiated a Fusarium wilt screening program selecting

${ }^{1}$ The majority of the contents of this paper has been published in Molecular Plant Pathology 8(3):333-341.

Proc. IS on Banana Crop Prot., Sust. Prod. 
somaclonal variants. A field selection from Giant Cavendish, known as 'GCTCV-218' (syn. 'Formosana'), eventually rescued the banana industry in Taiwan from destruction by Fusarium wilt (Hwang and Ko, 2004).

Non-conventional strategies, such as transformation, could be alternatives to conventional breeding. However, very few banana genes have been isolated and characterised to date, and the banana-Foc interaction has not been studied extensively at the molecular level. A molecular analysis of pathogen-induced genes may lead to a better understanding of resistance and may contribute to the development of biotechnological control strategies.

\section{MATERIALS AND METHODS}

Greenhouse trials to evaluate the resistance/tolerance of 'GCTCV-218' against Foc were conducted as described by Van den Berg et al. (2007). Briefly, 'Williams' (AAA genome, Cavendish subgroup) and 'GCTCV-218' plantlets were transferred into plastic cups containing water, and roots were gently crushed and inoculated by adding 2.5 $\mathrm{ml}$ of a Foc spore suspension $\left(10^{6}\right.$ conidia/ml of each isolate CAV 045, 092 an 105). Plants were maintained in the greenhouse until root sampling at $0,3,6,24$ and $48 \mathrm{~h}$ (for suppression subtractive hybridisation ( $\mathrm{SSH}$ ) and quantitative reverse transcriptase PCR (qRT-PCR)) and for a further 6 weeks until disease rating. Disease development in the greenhouse was evaluated using a modified version of the rating scale for Fusarium wilt of banana developed by Carlier et al. (2002) (Van den Berg et al., 2007). Three separate field trials were conducted in naturally infected soils in Mpumalanga, South Africa. Disease incidence was scored according to the presence (1) and absence (0) of external symptoms. Percentage disease severity for greenhouse and percentage disease incidence for field trials was calculated using the formula of Sherwood and Hagedorn (1958).

The banana SSH library was previously constructed and screened using a highthroughput DNA micro-array method. Clones were selected according to the Micro-array expression and Inverse Northern blot ratios (Van den Berg et al., 2004). The micro-array ratio was calculated as follows: Unsubtracted Tester ('GCTCV-218') / Unsubtracted Driver ('Williams') = antilog of (Expression Ratio 1 - Expression Ratio 2) in the base 2 (Van den Berg et al., 2004). The inverse Northern expression ratio was calculated as: density of tester/driver ('GCTCV-218'/'Williams') samples after normalisation of the data using an rDNA clone. Nucleotide sequences of the 79 selected SSH cDNA clones and sequence homologies were determined with BLAST programs (Altschul et al., 1990). The expression profiles of four putative defence-related genes, $P R-1, P R-3$ (AF 416677), $P A E$ and catalase 2, in 'GCTCV-218' and 'Williams' were assessed by qRT-PCR using LightCycler FastStart DNA Master ${ }^{\text {PLUS }}$ SYBR Green I (Roche Diagnostics) as described by Van den Berg et al. (2007).

Phenolics were extracted using a modified version of the method described by De Ascensao and Dubery (2003) (Van den Berg et al., 2007). Briefly, phenolics were extracted from $0.05 \mathrm{~g}$ root material with a $1-\mathrm{ml}$ solution $\mathrm{MeOH} / \mathrm{AC} / \mathrm{H}_{2} \mathrm{O}$. Supernatants were combined, concentrated to $1 \mathrm{ml}$ and used to determine the total soluble, free, $\mathrm{MeOH}$-soluble ester-bound and $\mathrm{MeOH}$-soluble glycoside-bound phenolic acids. The remaining precipitate was dried and used to extract the cell wall-bound phenolics. The Folin-Ciocalteau reagent was used to determine the phenolic content, and the concentration of phenols in the various extracts was calculated from a standard curve and expressed as $\mu \mathrm{g}$ Gallic acid/g dry weight.

Statistical analysis was conducted using General Linear Models (GLM) procedure of STATISTICA, version 7 (StatSoft Inc., 2004). Data from the disease rating and phenolic assays were analysed using a One-Way Analysis of Variance (ANOVA) and Tukey's Honest Significant Difference (HSD) test. Data from the qRT-PCR were analysed using ANOVA and Duncan's Multiple Range Test (DMRT). In all cases, significance was evaluated at $P<0.05$. 


\section{RESULTS}

Yellowing of banana leaves and wilting appeared 4 to 5 weeks after inoculation with Foc in the greenhouse. After 6 weeks, 'Williams' plants developed more severe internal symptoms than 'GCTCV-218' plants. Many of the 'Williams' plants scored the maximum value of 5 for internal symptoms compared to 1 (few) or 0 (no) internal symptoms in 'GCTCV-218'. Disease severity values for 'Williams' were 65 and $57 \%$ for the two trials compared to 34 and $38 \%$ in 'GCTCV-218' (Fig. 1A). No symptoms developed in the control plants of either 'GCTCV-218' or 'Williams'. Typical external symptoms appeared on 'Williams' plants as a yellowing of the leaf margins of older leaves. Severely infected banana plants showed the yellowing advancing from the oldest to the youngest leaves and the typical "skirt" of dead leaves hanging down around the pseudostem. Longitudinal splitting of the outer leaf bases of the pseudostem of susceptible plants was also often observed. In the field, 'GCTCV-218' consistently showed better tolerance to Fusarium wilt than 'Williams'. At the three test sites, 'GCTCV-218' plants had a disease incidence of 10, 34 and 14\% compared to 52, 76 and $72 \%$ for 'Williams' (Fig. 1B).

A cDNA library of 736 banana clones enriched for genes differentially expressed in 'GCTCV-218' at 6 hours post inoculation (hpi) of Foc race 4 was generated using SSH (Van den Berg et al., 2004). Subsequent high-throughput DNA micro-array screening resulted in the identification of 79 clones that were differentially expressed in 'GCTCV218 ' in response to Foc (Van den Berg et al., 2004) and these were sequenced. 55 of the 79 sequences showed significant homology to plant gene sequences while 24 had no significant homology to any sequences in databases. After sequence analysis and clustering, 15 non-redundant fragments were identified that were truly differentially expressed in the tolerant 'GCTCV-218' after Foc infection (Table 1). Micro-array expression and Inverse Northern Blot ratios correlated and were $\geq 1$, indicating that the fragments were up-regulated in response to Foc in 'GCTCV-218' compared to 'Williams'. They encode banana proteins with similarities to two putative peroxidases, two proteins of unknown function, a trypsin inhibitor, PR-1, a PAE, xylanase inhibitor, metallothionein, ribosomal protein $\mathrm{S} 3 \mathrm{a}$, response regulator 6 (putative role in twocomponent signal transduction), catalase 2, ferredoxin III, cytrochrome P450 and a putative senescence-associated protein.

The results from independent qRT-PCR experiments confirmed micro-array data and revealed a significant, differential induction of these genes in 'GCTCV-218' in response to Foc race 4 (Fig. 2). Catalase 2 expression was higher in the tolerant 'GCTCV-218' than in the susceptible 'Williams' by 6 hpi (Fig. 2A). PAE was upregulated in 'GCTCV-218' at 3 hpi while no up-regulation was observed in 'Williams'. Although the level of up-regulation is reduced, $P A E$ expression was higher in 'GCTCV218 ' than in 'Williams' at 3, 6, 24 and 48 hpi (Fig. 2B). Up-regulation of $P R-1$ occurred in both 'GCTCV-218' and 'Williams' (Fig. 2C). However, the expression ratio of $P R-1$ was more substantial after 3 and 6 hpi in 'GCTCV-218'. $P R-1$ expression was highest at 6 hpi in 'GCTCV-218', after which it was reduced. $P R-3$ was up-regulated in both 'GCTCV-218' and 'Williams' following inoculation with Foc. Maximal expression in 'Williams' occurred at $3 \mathrm{hpi}$, after which expression was reduced. However, in 'GCTCV218 ', $P R-3$ induction was highest at $6 \mathrm{hpi}$ and showed up-regulation throughout the time course after inoculation (Fig. 2D). PR-3 expression was higher in 'GCTCV-218' than in 'Williams' at 6, 24 and 48 hpi.

'GCTCV-218' produced a significantly higher total phenolic content when compared to 'Williams' at 0 and 6 hpi with Foc (Fig. 3A). Although there were no significant differences between the treatments, an induction in total phenolics was observed in 'GCTCV-218' after $6 \mathrm{~h}$, while induction in 'Williams' occurred only after 24 h. Total phenolic production in 'GCTCV-218' and 'Williams' were similar at 24 and 48 hpi. Free acids were significantly higher in 'GCTCV-218' when compared to 'Williams' at $0 \mathrm{~h}$, but decreased after inoculation in both cultivars. 'GCTCV-218' also had a significantly higher level of free acids at $24 \mathrm{hpi}$ (Fig. 3B). Although a significant decrease 
in glycoside phenolics occurred in 'GCTCV-218' 6 hpi, an increased induction was visible at 24 and $48 \mathrm{hrs}$ (Fig. 3C) while 'Williams' only responded at $48 \mathrm{hrs}$. Glycosidebound phenolics were significantly higher in 'GCTCV-218', 24 and $48 \mathrm{hrs}$ after inoculation when compared to 'Williams'. Ester-bound phenolics were expressed at similar levels in 'GCTCV-218' at 0 and 6 hpi, but a significant increase in content was observed at $24 \mathrm{hrs}$ and reached a plateau at $48 \mathrm{hpi}$ with Foc (Fig. 3D). The ester-bound phenolics in 'Williams', however, only increased at $48 \mathrm{hrs}$. 'GCTCV-218' responded to pathogen infection at 24 and 48 hpi with a significant increase in cell wall-bound phenolics compared to 'Williams'. In contrast, while containing a greater basal level compared to 'GCTCV-218' of phenolics prior to inoculation, phenolic content in 'Williams' decreased by 24 hpi (Fig. 3E).

\section{DISCUSSION}

Pathogenicity trials in the greenhouse and field showed that the Giant Cavendish somaclonal variant 'GCTCV-218' developed significantly less Fusarium wilt than the susceptible 'Williams' in South Africa, and can thus be considered tolerant to Foc 'subtropical race 4' (VCG-0120). 'Williams' plants developed typical Fusarium wilt symptoms both in the greenhouse and field. In the field, severely infected 'Williams' plants were entirely chlorotic, and, in some cases, large numbers of plants succumbed to the disease. This result has important implications for the continued cultivation of Cavendish banana in countries affected by Foc race 4. Hybrids with high levels of resistance to the pathogen are not always acceptable to Cavendish-dominated markets. However, the tolerant Cavendish banana selection 'GCTCV-218' could be considered a good replacement for susceptible Cavendish varieties in countries affected by Foc race 4 .

In this study, 15 non-redundant gene fragments associated with tolerance to Foc 'subtropical race 4' were identified in 'GCTCV-218'. Ten of the 15 clones showed significant similarities to defence-associated genes, indicating that the tolerant 'GCTCV$218^{\prime}$ recognises either Foc or virulence factors secreted by the pathogen and is able to respond at the transcriptional level through the induction of defence genes. Four defencerelated genes (catalase 2, $P A E, P R-1$ and $P R-3$ ) investigated in this study were significantly up-regulated in 'GCTCV-218'.

The induction of $P R-1$ and $P R-3$ (endochitinase) in 'GCTCV-218' following Foc infection, and the marked increase of $P R-1$ over time, suggests that proteins encoded by these genes are associated with plant defence in banana roots. The induction and greater accumulation of $P R-1$ in 'GCTCV-218' after Foc infection could play a role in the successful containment of the pathogen. This is consistent with the study of Pegg and Young (1982), who reported that the release of $\beta-1-3$ glucanase and chitinase might serve to inhibit Foc in banana. PR-1 proteins are the only family members for which no biochemical function is known. However, several studies indicate possible antifungal properties (Niderman et al., 1995). The association of PR-1 proteins with cell wall deposits implies a more structural role, possibly in the formation of physical barriers to prevent pathogen spread (Cutt and Klessig, 1992). PR-3 expression in 'GCTCV-218' could inhibit Foc growth due to biochemical degradation of fungal cell walls; it however could also possibly be linked to the systemic activation of defence responses away from the initial site of infection due to the their role in releasing elicitors. Chitinases are known to hydrolyse the chitin present in cell walls of fungi and can, therefore, serve as inhibitors of fungal growth (Collinge et al., 1993). Chitinases also release elicitor compounds from the pathogen or host cell walls, which in turn stimulates the defence system (Boller, 1987).

Catalase expression was significantly increased in 'GCTCV-218' at 6 and $48 \mathrm{hpi}$. This is consistent with the findings of García-Limones et al. (2002), who showed that catalase activities are enhanced in the incompatible interaction between chickpeas and $F$. oxysporum f. sp. ciceri. Consequently, they suggested that the expression of catalases in the roots is an early response to Fusarium infection. Catalases are known to decrease the levels of $\mathrm{H}_{2} \mathrm{O}_{2}$, which acts as a second messenger for the activation of plant-defence 
responses and has, in addition, been linked to cell-wall cross-linking and as a potential toxin against invading pathogens (Clark et al., 2000). Increased catalase activity may be a consequence of increased levels of $\mathrm{H}_{2} \mathrm{O}_{2}$ to protect the plant cells against the oxidative burst.

The up-regulation of $P A E$ in 'GCTCV-218' found in this study is most likely related to the modification of the pectin component in root cell walls, which in turn may affect cell wall strengthening. PAEs catalyse the de-acetylation of pectin, which is a major compound of plant primary cell walls (Vercauteren et al., 2002). More specifically, PAE hydrolyses acetyl esters in the homogalacturonan regions of pectin, thereby modifying cell walls during root development and pathogen interactions (Savary et al., 2003). PAE has also been demonstrated to be up-regulated in Arabidopsis thaliana roots shortly after nematode infection (Vercauteren et al., 2002).

Based on the fact that some differentially expressed genes isolated from ' $\mathrm{GCTCV}$ 218' (including PAE and peroxidase genes; Table 1) are implicated in cell wall strengthening, we investigated the phenolic content in 'GCTCV-218' and 'Williams' in response to Foc and found a significantly higher level of total peholics and an increase in cell wall-bound phenolics in 'GCTCV-218'.

The higher levels of total soluble phenolics in 'GCTCV-218' compared to 'Williams' observed in this study suggest that constitutive defence compounds are present in higher concentrations in tolerant banana clones, even before contact with the pathogen. This increased presence of phenolics could contribute to an enhanced plant response after infection. In this study, ester-bound phenolics were expressed at increased levels in 'GCTCV-218', 24 hrs after pathogen attack. Evidence strongly suggests that esterification of phenols to cell wall materials, is a common theme in the expression of disease resistance (Fry, 1987). The early increase of glycoside-bound phenolics in 'GCTCV-218' may further contribute to disease tolerance by being toxic to the pathogen and thereby preventing pathogen spread. The role of phenolics in defence responses in banana has been illustrated previously. De Ascensao and Dubery (2000) reported a substantial increase in total soluble phenolics in banana roots of 'FHIA-01' (AAAB genome, syn. 'Goldfinger') 8 hours after treatment with elicitors from Foc race 4. This is in contrast to 'Williams', which responded only after $12 \mathrm{hrs}$ and did not show the same prominent increase in phenolics that were found in the tolerant hybrid. Phenolics are precursors of several secondary metabolites involved in disease resistance, such as phytoalexins and lignin (Matern et al., 1995). In this study, 'GCTCV-218' had a greater capacity for phenolic metabolism in response to Foc and it also displayed a high basal level of total soluble phenolics, free acids and glycoside-bound phenolics before pathogen infection. Strengthening of cell walls is generally accomplished by infusion of phenolics into, or the apposition of phenolic-containing material against, the cell wall (Beckman et al., 1974; Ride, 1975). The constitutive presence of phenolics suggests that 'GCTCV-218' has preexisting defence mechanisms that protect the plant. 'GCTCV-218' responded strongly to pathogen attack by producing high levels of cell wall-bound phenolics. This suggests that cell wall strengthening and subsequent lignin deposition is taking place. The high level of phenolics found in 'GCTCV-218' may also possibly contribute to the effective and timeous production of papillae and gels in response to Foc. 'GCTCV-218' is able to actively induce a structural and biochemical defence response against Foc. Apart from simply inducing strong defence responses, 'GCTCV-218' appears to be able to induce them early enough to contain Foc and to prevent further spread.

An effective resistance response against Fusarium wilt diseases depends on the rate and extent of recognition and activation of the defence mechanisms (Beckman, 1987, 1990). 'GCTCV-218' showed that it is able to respond rapidly to Foc infection by inducing genes involved in biochemical and structural defence mechanisms. Two genes in this study, $P R-1$ and $P A E$, were induced very early $(3 \mathrm{hpi})$ in the tolerant defence response, while $P R-3$ and catalase 2 followed with a significant induction at 6 hpi. In 'Williams', $P R-1$ was induced by 3 hpi, showing that $P R-1$ is also induced in this cultivar, but more slowly and to a lesser degree than in 'GCTCV-218'. 
This study has provided information on a tolerant plant-pathogen interaction and a soil-borne root pathogen. The results shed light on genes involved in defence and provide a step towards understanding Fusarium wilt of banana and thereby developing an effective disease management strategy.

\section{ACKNOWLEDGEMENTS}

We thank THRIP of the Department of Science and Technology, the National Research Foundation, the Banana Growers' Association of South Africa and the Mellon Foundation (USA) for funding, and Du Roi Laboratories for supplying tissue-cultured plants.

\section{Literature Cited}

Altschul, S.F., Gish, W., Miller, W., Myers, E.W. and Lipman, D.J. 1990. Basic local alignment search tool. J. Mol. Biol. 215:403-410.

Beckman, C.H. 1987. The nature of wilt diseases of plants. American Phytopathological Society, St Paul, Minnesota, USA.

Beckman, C.H. 1990. p.93-105. In: R.C. Ploetz (ed.), Fusarium Wilt of Banana. St Paul, Minnesota, APS Press, USA.

Beckman, C.H., Mueller, W.C. and Mace, M.E. 1974. The stabilization of artificial and natural cell wall membranes by phenolic infusion and its relation to wilt disease resistance. Phytopathology 64:1214-1220.

Boller, T. 1987. Hydrolytic enzymes in plant disease resistance. In: T. Kosuge and F.W. Nester (eds.), Plant-Microbe Interactions, Molecular And General Aspects. Vol. 2. MacMillan, New York.

Carlier, J., De Waele, D. and Escalant, J.V. 2002. Global Evaluation Of Musa Germplasm For Resistance To Fusarium Wilt, Mycosphaerella Leaf Spot Diseases And Nematodes. INIBAP Technical Guidelines 6, Montpellier, France.

Clark, D., Durner, J. Navarre, D.A. and Klessig, D.F. 2000. Nitric oxide inhibition of tobacco catalase and ascorbate peroxidase. Mol. Plant Microbe Interac. 13:1380-1384.

Collinge, B., Kragh, K.M., Mikkelsen, J.D., Nielsen, K.K., Rasmussen, U. and Vad, K. 1993. Plant chitinases. Plant J. 3:31-40.

Cutt, J.R. and Klessig, D.F. 1992. Pathogenesis-related proteins. In: T. Boller and F. Meins (eds.), Plant Gene Research: Genes Involved In Plant Defence. SpringerVerlag, Wien, Austria.

De Ascensao, A.R.D.C.F. and Dubery, I.A. 2000. Panama disease: Cell wall reinforcement in banana roots in response to elicitors from Fusarium oxysporum f. sp. cubense race 4. Phytopathology 90:1173-1180.

De Ascensao, A.R.D.C.F. and Dubery, I.A. 2003. Soluble and wall-bound phenolics and phenolic polymers in Musa acuminata roots exposed to elicitors from Fusarium oxysporum f. sp. cubense. Phytochemistry 63:679-686.

Fry, S.C. 1987. Intracellular feruloylation of pectic polysaccharides. Planta 171:205-211.

García-Limones, C., Hervás, A., Navas-Cortés, J.A., Jiménez-Díaz, R.M. and Tena, M. 2002. Induction of an antioxidant enzyme system and other oxidative stress markers associated with compatible and incompatible interactions between chickpea (Cicer arietinum L.) and Fusarium oxysporum f. sp. ciceris. Physiol. Mol. Plant Pathol. 61:325-337.

Hwang, S. and Ko, W. 2004. Cavendish banana cultivars resistant to Fusarium wilt acquired through somaclonal variation in Taiwan. Plant Dis. 88:580-588.

Hwang, S. and Tang, C. 1996. p.67-75. In: E. Frison, J.P. Horry and D. De Waele (eds.), New Frontiers In Resistance Breeding For Nematode, Fusarium And Sigatoka. INIBAP, France.

Matern, U., Grimmig, B. and Kneusel, R.E. 1995. Plant cell wall reinforcement in the disease-resistance response: molecular composition and regulation. Can. J. Bot. 73:S511-S517.

Niderman, T., Genete, I., Bruyere, T., Gees, R., Stintzi, A., Legrand, M., Fritig, B. and 
Mosinger, E. 1995. Pathogenesis-related PR-1 proteins are antifungal: Isolation and characterization of three 14-kilodalton proteins of tomato and of a basic PR-1 of tobacco with inhibitory activity against Phytophthora infestans. Plant Physiol. 108:1727.

Pegg, G.F. and Young, D.H. 1982. Purification and characterization of chitinase enzymes from healthy and Verticillium albo-atrum- infected tomato plants and from $V$. alboatrum. Physiol. Plant Path. 21:389-409.

Ploetz, R.C. 2005. Panama disease, an old nemesis rears its ugly head. Part 2, The Cavendish era and beyond. APSnet Feature, October 2005.

Ride, J.P. 1975. Lignification in wounded wheat leaves in response to fungi and its possible role in resistance. Physiol. Plant Pathol. 5:125-134.

Robinson, J.C. 1996. Banana and Plantain. Wallingford, CAB International.

Savary, B.J., Nunez, A., Liu, L.S. and Yoo, S. 2003. Pectin Acetylesterase - Analysis and Application for Sugar Beet Pectin utilization. Proc. First Joint International Beet Research - American Society of Sugar Beet Technologists Congress. Beet Sugar Development Foundation, Denver, Co.

Sherwood, R.T. and Hagedorn, D.J. 1958. Determining common root rot potential of pea fields. Wis. Agric. Exp. Stat. Bull. 531:12.

van den Berg, N., Crampton, B.G., Hein, I., Birch, P.R.J. and Berger, D.K. 2004. Highthroughput screening of suppression subtractive hybridization cDNA libraries using DNA microarray analysis. BioTechniques 37:818-824.

van den Berg, N., Hein, I., Birch, P.R.J., Berger, D.K., Wingfield, M.J. and Viljoen, A. 2007. Tolerance in banana to Fusarium wilt is associated with early up-regulation of cell wall-strengthening genes in the roots. Mol. Plant Path. 8(3):333-341.

Vercauteren, I., de Almeida, E.J., De Groodt, R. and Gheysen, G. 2002. An Arabidopsis thaliana pectin acetylesterase gene is up-regulated in nematode feeding sites induced by root-knot and cyst nematodes. Mol. Plant-Microbe Interac. 15:404-407.

Viljoen, A. 2002. The status of Fusarium wilt (Panama disease) of banana in South Africa. S. Afr. J. Sci. 98:341-344. 


\section{$\underline{\text { Tables }}$}

Table 1. BLASTX identities of non-redundant clones derived from the banana Suppression Subtractive Hybridisation (SSH) library enriched for transcripts induced in roots of Cavendish selection 'GCTCV-218' (AAA) after infection with Fusarium oxysporum f. sp. cubense 'subtropical race 4' compared to 'Williams' (AAA).

\begin{tabular}{|c|c|c|c|c|c|c|c|c|}
\hline Putative identity & $\begin{array}{c}\text { Accession no. } \\
\text { of best BlastX } \\
\text { hit }\end{array}$ & Species & $\begin{array}{c}\text { E- } \\
\text { value }\end{array}$ & Functional category & $\begin{array}{l}\text { Accession no. } \\
\text { of SSH clone }\end{array}$ & $\begin{array}{l}\text { No. of } \\
\text { clones }\end{array}$ & $\begin{array}{l}\text { Micro - } \\
\text { array } \\
\text { ratio }^{\mathrm{a}}\end{array}$ & $\begin{array}{c}\text { Inverse } \\
\text { Northern Blot } \\
\text { ratio }^{\mathrm{b}}\end{array}$ \\
\hline Catalase 2 & AAG61140.2 & Zantedeschia aethiopica & $5 \mathrm{e}^{-50}$ & Protection against oxidative burst & DQ 531612 & 1 & 1.2 & 2.1 \\
\hline Ferredoxin III & $\mathrm{P} 27788$ & Zea mays & $3 e^{-45}$ & Energy metabolism & DQ 531614 & 1 & 1.1 & 1.4 \\
\hline Metallothionein & AAG44757.1 & Musa acuminata & $4 e^{-27}$ & Cell rescue/defence & DQ 531613 & 2 & 1.0 & 1.2 \\
\hline Pectin acetylesterase & BAC 07121.1 & Oryza sativa & $6 \mathrm{e}^{-92}$ & Pectin modification & DQ 531615 & 2 & 1.1 & 2.0 \\
\hline Peroxidase & AB 013389 & Arabidopsis thaliana & $2 \mathrm{e}^{-05}$ & Secondary metabolism - Lignin biosynthesis & DQ 531609 & 2 & 1.7 & ND \\
\hline Peroxidase & BAB 19339.1 & Oryza sativa & $6 \mathrm{e}^{-49}$ & Secondary metabolism - Lignin biosynthesis & DQ 531618 & 8 & 1.6 & 1.2 \\
\hline PR-1 & AAC 25629.1 & Zea mays & $4 e^{-17}$ & In-vitro antifungal activity (defence) & DQ 531622 & 2 & 1.0 & ND \\
\hline $\begin{array}{l}\text { Putative senescence } \\
\text { associated protein }\end{array}$ & BAB33421.1 & Pisum sativum & $4 e^{-43}$ & Unknown & - & 2 & 1.0 & ND \\
\hline Response regulator 6 & BAB 20581.1 & Zea mays & $7 e^{-56}$ & Signal transduction & DQ 531611 & 1 & 1.0 & ND \\
\hline Ribosomal protein S3a & CAD 56219 & Cicer arietinum & $2 \mathrm{e}^{-42}$ & Protein synthesis & DQ 531623 & 1 & 1.2 & 3.1 \\
\hline Trypsin inhibitor & CAA 29122.1 & Vigna unguiculata & $3 \mathrm{e}^{-04}$ & Protease inhibitor & DQ 531617 & 4 & 1.3 & 6.9 \\
\hline Unknown protein & CAE 02910 & Oryza sativa & $9 \mathrm{e}^{-12}$ & Unknown & DQ 531616 & 4 & 1.1 & ND \\
\hline Unknown protein & AAL 77110 & Hordeum vulgare & $4 e^{-45}$ & Unknown & DQ 531610 & 4 & 1.0 & ND \\
\hline $\begin{array}{l}\text { Unspecific mono- } \\
\text { oxygenase, cytochrome } \\
\text { P450 }\end{array}$ & T02995 & Nicotiana tabacum & $2 e^{-41}$ & Phenylpropanoid biosynthesis & - & 5 & 1.3 & 1.0 \\
\hline Xylanase inhibitor & CAD 27730.1 & Triticum aestivum & $1 \mathrm{e}^{-34}$ & Cell rescue/defence & DQ 531620 & 2 & 1.2 & 1.7 \\
\hline
\end{tabular}

${ }^{\text {a }}$ Micro-array expression ratio: Unsubtracted Tester ('GCTCV-218') / Unsubtracted Driver ('Williams') = antilog of (Expression Ratio 1 - Expression Ratio 2) in the base 2 (Van den Berg et al., 2004)

b The inverse Northern expression ratio was calculated as follows: density of "tester"/"driver" ('GCTCV-218'/'Williams') samples after normalisation of the data using an rDNA clone (Van den Berg et al., 2004)

$\mathrm{ND}=$ not determined 


\section{Figures}

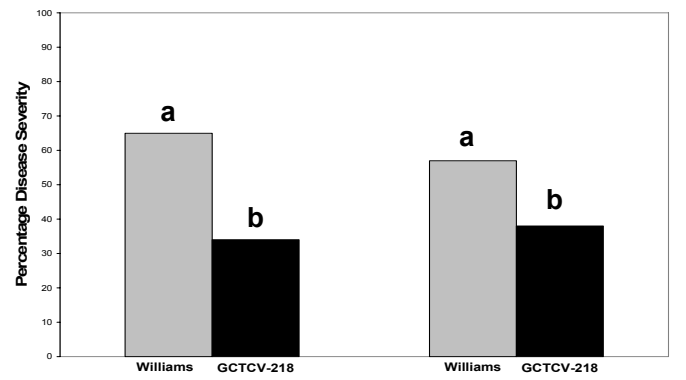

A.

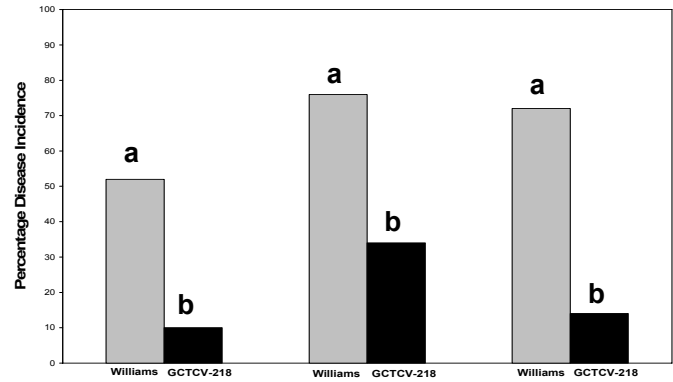

B.

Fig. 1. Cavendish banana selection 'GCTCV-218' (AAA) showed increased tolerance to Fusarium oxysporum f. sp. cubense (Foc) 'subtropical race 4' compared to 'Williams' (AAA). (A) Percentage disease severity of 'Williams' (grey) and 'GCTCV-218' (black) infected with Foc during two independent greenhouse trials. (B) Percentage disease incidence during three independent field trials in Foc-infected areas in South Africa were calculated using the formula of Sherwood and Hagedorn (1958). Data were analysed using ANOVA and the Tukey Honest Significant Difference Test. Bars presented with the same letter are not significantly different at $P<0.05$.

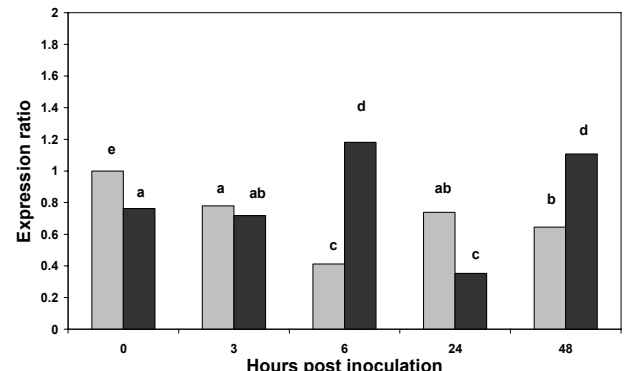

A.

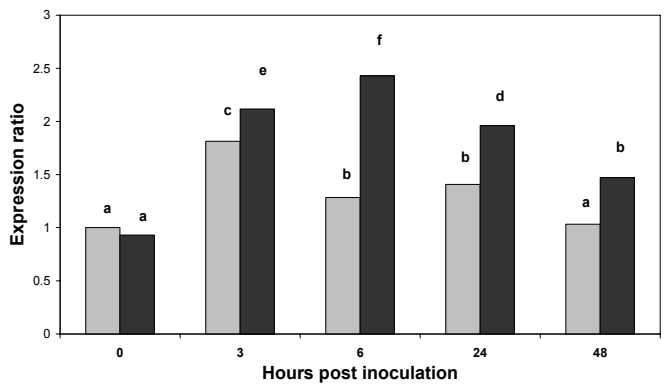

C.

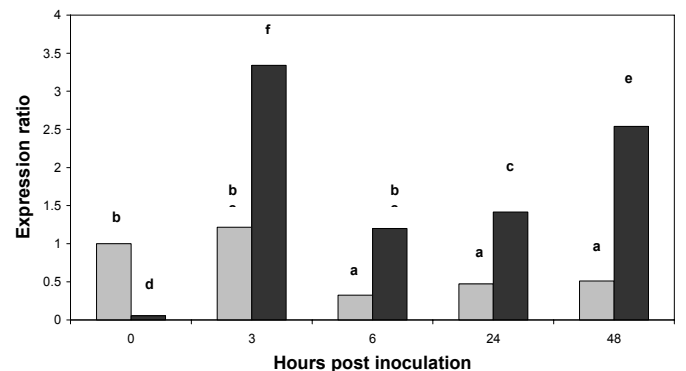

B.

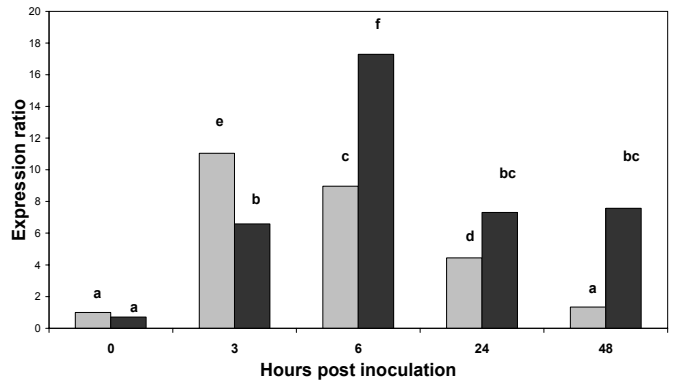

D.

Fig. 2. Cell wall strengthening and defence-related genes are induced to a greater extent in Cavendish selection 'GCTCV-218' (AAA) (black) after infection with Fusarium oxysporum f. sp. cubense (Foc) 'subtropical race 4' compared to 'Williams' (AAA) (grey). Relative gene expression levels at 0, 3, 6, 24 and 48 hpi of (A) catalase 2, (B) pectin acetyl esterase, (C) PR-1 and (D) PR-3 in roots of 'Williams' and 'GCTCV-218' bananas after infection. Expression ratios were calculated by quantitative RT-PCR and expressed relative to the transcripts in 'Williams' roots at 0 hpi. Data were analysed using ANOVA and the Duncan's Multiple Range Test. Bars presented with the same letter are not significantly different at $P<0.05$. 


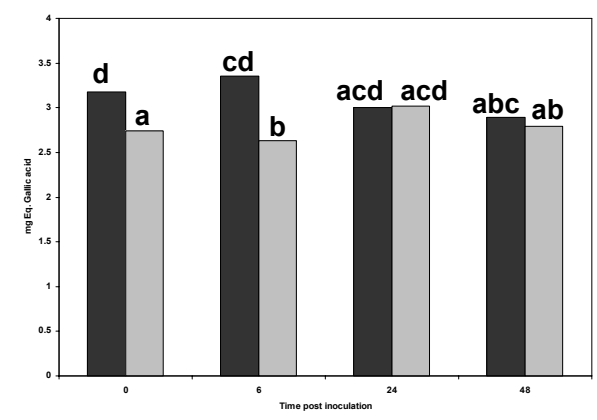

A.

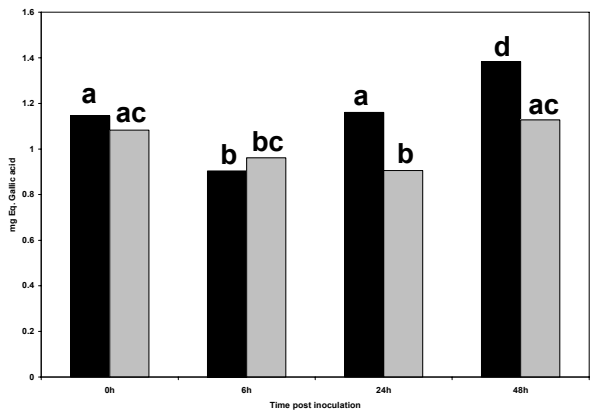

C.

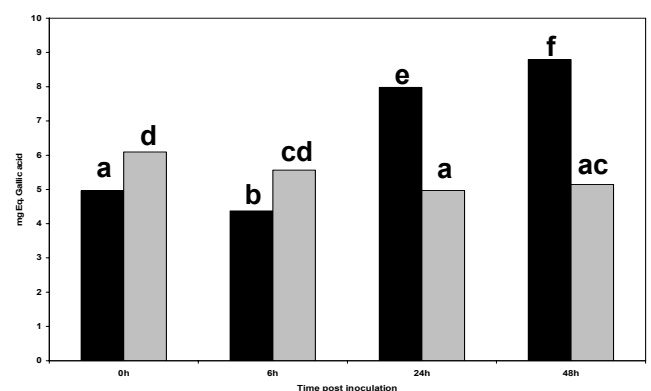

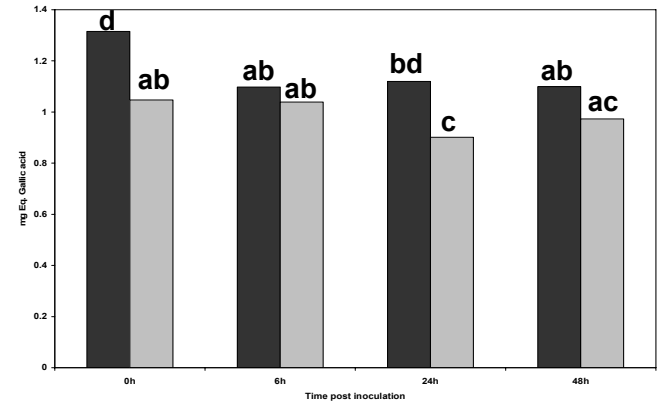

B.

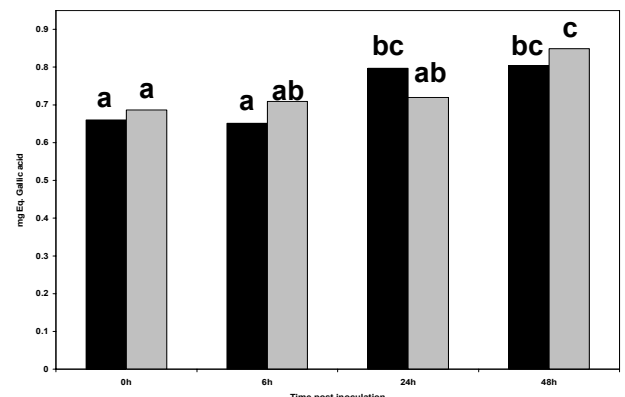

D.

E.

Fig. 3. (A) Total phenolic content in Cavendish banana selection 'GCTCV-218' (AAA) (black bars) is significantly higher at 0 and $6 \mathrm{~h}$ post infection with Fusarium oxysporum f. sp. cubense (Foc) 'subtropical race 4' compared to 'Williams' (AAA) (grey bars). (B) Basal levels of free acids are significantly higher in 'GCTCV-218' compared to Williams at 0h. (C) Glycoside-bound phenolics were significantly higher in 'GCTCV-218', 24 and 48 hpi compared to 'Williams'. (D) Ester-bound phenolics were expressed at similar levels in 'GCTCV-218' at 0 and $6 \mathrm{hrs}$ after inoculation, but a significant increase in content was observed at $24 \mathrm{hrs}$ after Foc infection. (E) Cell wall-bound phenolic compounds are significantly increased in roots of 'GCTCV-218' after infection with Foc 'subtropical race 4' compared to 'Williams'. Total phenolics and cell wall-bound phenolics were extracted from roots of 'Williams' and 'GCTCV-218' and determined with the Folin reagent as milligrams of Gallic acid per gram dry weight. Data were analysed using ANOVA and the Tukey Honest Significant Difference Test. Bars presented with the same letter are not significantly different at $P<0.05$. 